\title{
Survey on Design and Analysis of Mobile IP
}

\author{
Barenya Bikash Hazarika \\ Department of CSE \& IT \\ Assam Don Bosco University
}

\author{
Bobby Sharma \\ Department of CSE \& IT \\ Assam Don Bosco University
}

\begin{abstract}
In today's world, communication is the most pervasive trend [13]. Among them, mobile communication is the most popular type of communication. Mobile communication services are increasing remarkably and among them mobile services that provides Internet access from mobile terminals are increasing day by day. Mobile IP is one of the dominating protocols which provides the support of mobility in the internet. [4] It represents a simple and scalable global mobility solution. In this paper, the analysis of mobile IP is presented. After the protocol overview, we then proceed to brief current developments namely Mobile IPv4 and Mobile IPv6, and the current state of standardization of Mobile IP.
\end{abstract}

\section{Keywords}

MIP, MIPv4, MIPv6, DHCPv6, Home Agent, Foreign Agent, Mobile Node, Care of Address, Correspondent Host.

\section{INTRODUCTION}

Mobile IP is a standard communication protocol of Internet Engineering Task Force (IETF) standard that is designed to give the mobile device users the freedom to move from a home network to a foreign network while maintaining a permanent IP address. [6] A mobile node can change its location and still access and communicate with and through the computer's home network. Assigning a different IP address to the mobile node is cumbersome. Thus, under the current Internet Protocol the mobile node loses routing if it moves without changing its address. If the mobile node does change the address, it loses connection. Mobile IP solves this problem by giving the mobile node the freedom to use two IP addresses, the first address, which is the fixed home address, gives the node a permanent home address and the second address, which is a care of address, change at each new point of attachment. [18] Mobile IP enables a computer to roam freely on the internet or handles mobility. [15] The following figure illustrates the general Mobile IP topology.

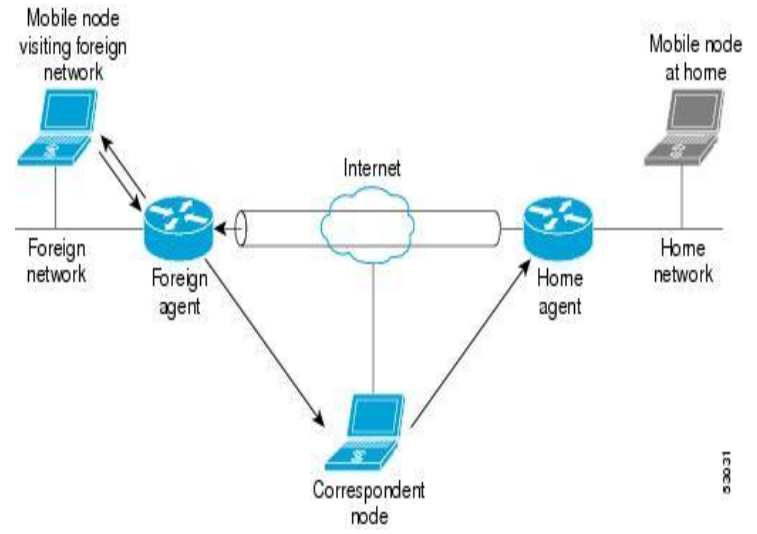

Fig 1- Mobile IP topology (source:google images)

\section{OVERVIEW OF MOBILE IP}

The Mobile IP or the MIP is the internet protocol (IP) which was developed by IETE. Mobile IP (MIP) was first proposed to support user host mobility on the internet. [19] It is probably the oldest and most widely known mobility management proposal. Generally MIP is more useful where wireless technology is used. It works by making it appear to the rest of the Internet as if the mobile node were always addressable at its home network. [20]

In MIP, the home address of the mobile node always identifies the mobile node. [1] When away from a home, a care of address is associated to the mobile node with its home address by providing information about the mobile node $(\mathrm{MN})$ 's present attachment point to the internet or an organizational network. [18] MIP uses a special mechanism called registration to register care of address with a home agent. [21] As a whole MIP can be thought of cooperation of 3 major subsystems-

- Firstly, there is a mechanism of discovery, where, the mobile computers determines their new attachment points. [22]

- Secondly, when the mobile computer knows the new attachment point and its IP address, it registers an agent with its home network.[22]

- Lastly, MIP defines simple mechanisms to deliver datagrams to mobile node when it is away from its home network.[9]

\subsection{Main Components Of Mobile Ip}

Let us assume that a mobile node is connected to a mobile network. The network provided by the service provider is called the Home Network and the address of it is called as home address. The Networks other than this network at which mobile node can connect is called Foreign Network and the network to which the mobile node is currently connected is called Visited Network. Mobile IP introduces the following new functional entities. [23]

Mobile Node--A host that changes its address frequently from one network to another without changing its IP addresses. [18]

Home agent-- A router on the home network of a mobile node which delivers data packets to the departed mobile nodes and maintains present location information for each. [18]

Foreign agent--It stores information about mobile nodes visiting its network. For datagrams sent by a mobile network, the FA may serve as a default router for registered Mobile networks. [23] Mobile IP uses care-of-address which are advertised by the foreign agents. If there foreign agents available in the host network, the MN has to search for an address and advertising it. 


\subsection{Basic Operations Of Mobile Ip}

Mobile IP is a way of performing three related operations-

- Agent advertisement and discovery- Mobility agents advertise their availability. The operation involves broadcast of advertisements by the routers to their connected sub networks. [22] Mobility agents employ ICMP router advertisement messages (RFC 1256), to carry the necessary information. [18] This is done by defining new extensions for the basic ICMP messages in a way that doesn't disturb existing entries that may be using Router advertisement for its originally specified purposes. In the modified advertisements transmitted by home agents and foreign agents, the mobile node finds information specifying the following configuration information:

- Whether the mobility agent is a home agent, a foreign agent, or both. [22]

- How long the care of address may be used by the mobile node.

- How frequently the mobile node should expect to hear advertisement from the agent. [22]

- Whether the foreign agent has rebooted since the last time the mobile node registered. [14]

- Registration- If the mobile node is not in its home network, it registers its care-of-address with its home agent. [5] The mobile node initiates the registration process.It sends a Registration Request to the foreign network, which consist of the mobile node's home address, the care-of address, the home agent's address etc. Once a mobile node discovers a care of address, it sends the information to its home agent. The home agent will be then able to redirect appropriate traffic to the mobile node. [22] The process by which the mobile node send its care of address to the home agent is called registration.

- Tunnelling-Once the mobile node has reported its care of address to the home agent, the problem of supporting seamless connectivity is simplified to the problem of delivering all packets from the home address to the mobile node at its care-of address, unchanged its final form. [22] The problem can be solved by tunnelling the packet i.e. the home agent has to tunnel the diagrams to the care-of address.

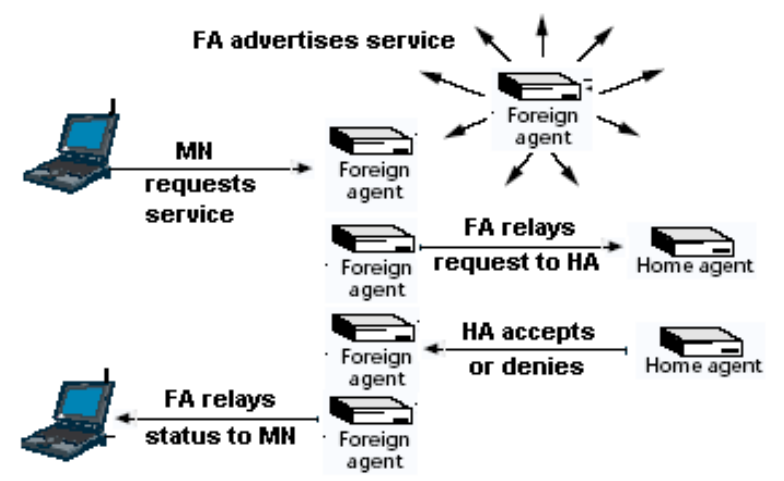

Fig 2-Registration overview (source:google images)

\subsection{Route Optimization Of Mobile Ip}

MIP enables a mobile host to send datagrams to the correspondent host directly, routed by its home agent and foreign agent. [24] The basic idea behind route optimization is that the routes to the mobile nodes from their corresponding nodes can be improved if the correspondent node has an updated routing table. [24] With an updated binding, the correspondent node will be able to send the encapsulated datagrams directly to the node's care off address instead of relying on a possibly distant home agent to do so.

Mobile IP enables a mobile host (MH) to send the data packets to the correspondent node directly, routed by its home agent (HA) and foreign agent (FA). Home agents intercepts the datagrams through a IPsec tunnel to the correspondent node. [11]

Packets destined to $\mathrm{MH}$ are often are routed along a longer path than optimal. The redundant routing in mobile IP is called "triangle routing". [10]

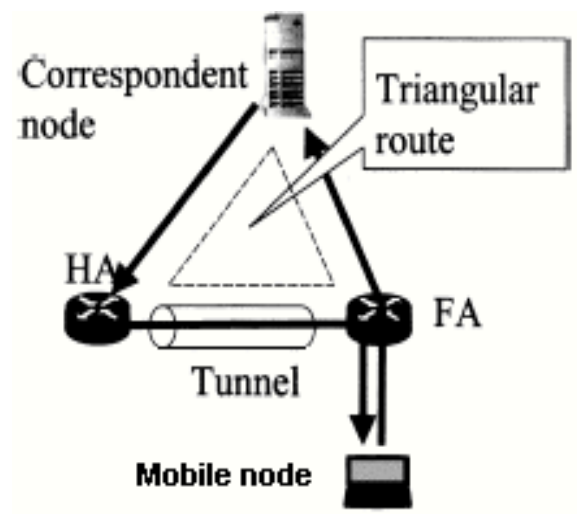

Fig 3-Traingle routing (source:google images)

In order to deliver bindings to the correspondent nodes of the mobile node, route optimization defines four new messages sent to the same port as the base Mobile IP.

- Binding warning-The Mobile node informs its correspondent node to get a new binding. It informs the recipient that the target node might get benefitted by obtaining a new binding for the mobile node. [22] Usually the recipient is the home agent (HA).

- Binding request-Correspondent node asks for new request. A corresponding node can anytime determine that its binding is stale, or is going stale. [5] The corresponding node sends a 64 bit number to the HA for use in protecting against replay attacks.

- Binding update-Correspondent node receives a new binding. The HA sends a binding update message to those corresponding nodes that need them. The binding update must be accompanied by the route optimization authentication extension. [13]

- Binding acknowledgement-Corresponding node receives a new binding. The binding acknowledgement message is used to acknowledge the reception of binding. The 64 bit identification field, again, protects against replays and allows the acknowledgement to be associated with a pending binding update. [22] 


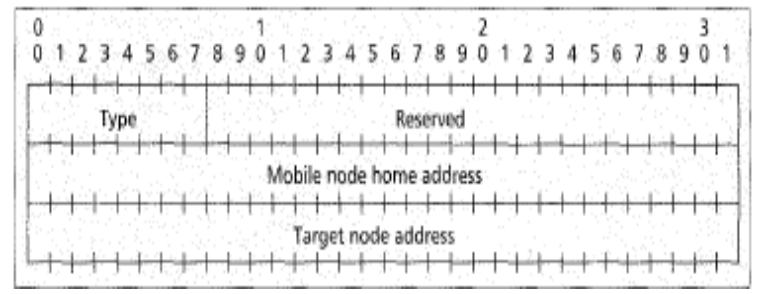

Fig 4: Binding Warning (source:google images)

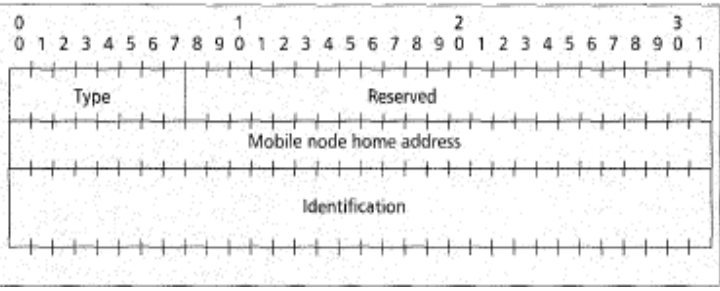

Fig 5: Binding Request (source:google images)

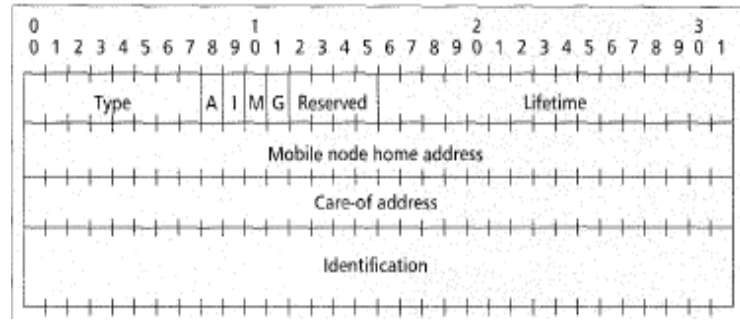

Fig 6: Binding Update (source:google images)

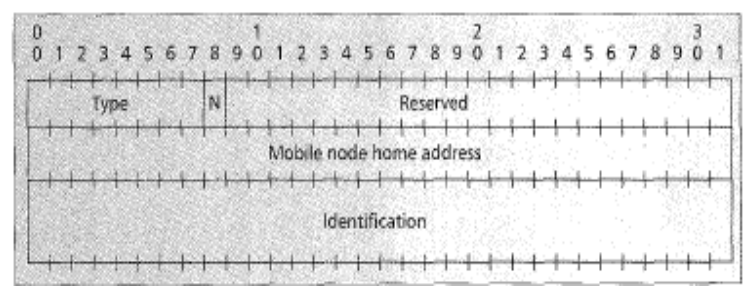

Fig 7: Binding Acknowledgement (source:google images)

\section{PROTOCOLS OF MOBILE IP 3.1 Mobile Ipv4 (Mipv4)}

MIPv4 assumes that a node IP address uniquely identifies the node's point of attachment to the internet. [5] Two mechanisms take place for a node to change its point of attachment:

1. The node will have to change its IP address in order to change its point of attachment. [10]

2. 2.Host specific routes, must be propagated throughout much of the Internet routing fabric. [25]

Operations of MIPv4 include 3 main steps

Agent Discovery: The mobile agent send packets to announce that they are present on each route where it provides services. Agent advertisement are sent periodically. [6]

\begin{tabular}{|c|c|c|c|c|c|}
\hline Vers=4 & $\mathrm{IHL}$ & Type of Service & \multicolumn{2}{|c|}{ Total Length } & \multirow{3}{*}{$\begin{array}{l}\text { IP Header } \\
\text { [RFC 791] }\end{array}$} \\
\hline \multicolumn{3}{|c|}{ Identification } & Flags & ent Offset & \\
\hline Time & live & ProtocoHCMP & Header & cksum & \\
\hline & Source & ess $=$ home ard/or & oreign agent's address & this link & \multirow{8}{*}{$\begin{array}{c}----- \\
\text { ICMP } \\
\text { Router } \\
\text { Advertisement } \\
\text { [RFC 1256] }\end{array}$} \\
\hline \multicolumn{5}{|c|}{ 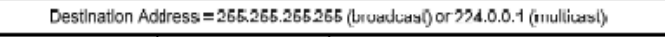 } & \\
\hline & & Code & Che & & \\
\hline Num & ddrs & Addr Entry Gize & Lifetime (of thi & (vertisement) & \\
\hline \multicolumn{5}{|c|}{ Router Addiess [1] } & \\
\hline \multicolumn{5}{|c|}{ Preference Level [1] } & \\
\hline \multicolumn{5}{|c|}{ Router Address [2] } & \\
\hline \multicolumn{5}{|c|}{ Preference Level [2] } & \\
\hline \multicolumn{5}{|c|}{$\ldots$} & \multirow{6}{*}{$\begin{array}{l}------ \\
\text { Mobility Agont } \\
\text { Advertisement } \\
\text { Extension } \\
\text { [RFC 2002] }\end{array}$} \\
\hline Typ & $=16$ & Length & Sequen & umber & \\
\hline (ma & mum) $\mathbf{R}$ & ration Lifetime & 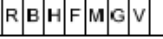 & Reserved & \\
\hline \multicolumn{5}{|c|}{ Care-of-Address [ $[1]$} & \\
\hline \multicolumn{5}{|c|}{ Care-ofinddress [2] } & \\
\hline \multicolumn{5}{|c|}{$\ldots$} & \\
\hline
\end{tabular}

Fig 8: Agent advertisement messages (source:google images)

\section{Agent Registration}

When we move away from the original network, MN must register the address to HA to make sure that the HA will be able to forward packets to MN exactly to MN. [6] Depending on the method associated with FA, MN can registrar directly with the HA or indirectly via FA.

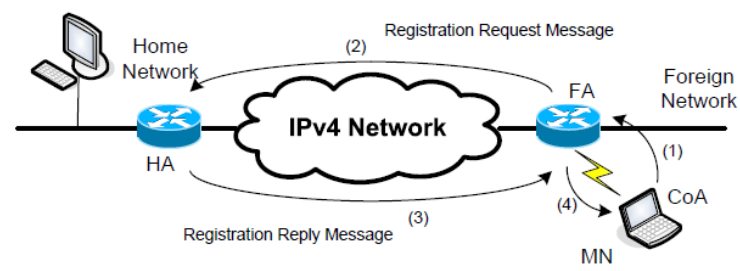

Fig 9: COA registration

\section{Data transfer}

After registering carefully, HA encapsulates each of packets with MN's COA and forwards them to the MN's current location via its FA.

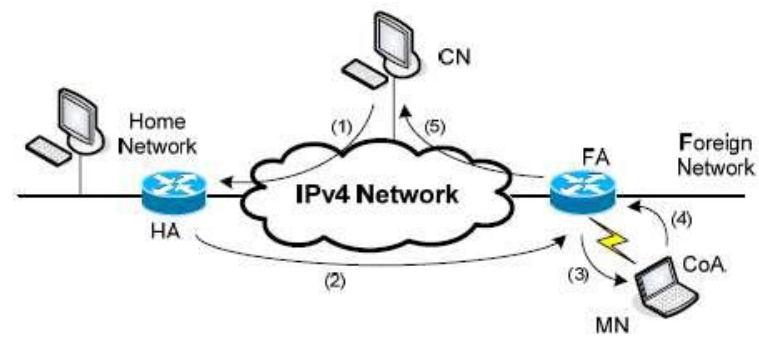

Fig 10: Data transfer

Issues of MIPv4

The main issue of MIPv4 is in routing. It suffers a long handover delay due to triangular routing because, the mobile node uses its present address in the field of source address. It also has the issue of large signalling overhead due to registration updates. MIPv4 also has the problem of reverse filtering. [16] 


\section{Solution of MIPv4}

Route optimization was proposed to solve the triangular routing problem. By using route optimization a mobile node can communicate with a corresponding node directly, bypassing the home agent completely. [7] Message from the $\mathrm{CN}$ are directly routed to the MN's COA without passing through the HA. It should be made compulsory for each station to maintain a binding cache, which includes CoA of $\mathrm{MN}$. Otherwise $\mathrm{CN}$ sends the datagrams to HA which tunnels them to MN

Ingress filtering can be solved by using Montenegro, G.

\subsection{Mobile Ipv6(Mipv6)}

MIPv6 is based on experience gained from MIPv4. It was expected to replace the MIPv4. [17] Most of its components are similar to that of MIPv4. [5] Operation of MIPv6 include four steps :CoA creation, $\mathrm{CoA}$ registration, Movement detection and Data delivery.

In mobile IPv6,the mobile node should assign three IPv6 addresses-

1. Home address: It is permanently assigned to the mobile node.

2. Current link local address: It gives the link of the current HA.

3. CoA of the MN: It is associated to the mobile node while visiting a particular foreign subnet.

MN's CoA is co-located CoA in MIPv6. It allows the MN to encapsulate and decapsulate packets and also it connects to the HA directly on any foreign link without notifying FA as the FA function is not there in MIPv6. [8] When the MN moves from one network to another, CoA is automatically allocated to it in the foreign network for its features namely:

Stateless: MN combines IPv6-prefix and its MAC address and creates a new IPv6 address, which is different from other addresses.

Statefull: MN contacts the local router by sending a CoA message. The local router allocates a new IPv6 address to MN by using DHCPv6.

The HA keeps a binding between the MN's CoA and its HA.The ce data structure which is centrally collected by each node is used as binding cache. [8] In MIPv6, a procedure known as DAD(Duplicate Address Detection) is called on to determine the uniqueness of the new MN's CoA inwhich a Mn sends sends a neighbour silicitation message with a set timer to ask that its address is being used or not. [8]If no replies then $\mathrm{MN}$ can assume that the node is unique in the network and could use the address.

\section{Issues of MIPv6}

- Security issues: The mobile node using IPv6, has no commonly deployed mechanism that a mobile node can present its credentials.[3] Secure route optimization technique is used to enhance the performance in IPv6. Route optimization means that when a mobile node receives the tunnelled message, it informs corresponding node about its present care of address by sending a binding update message. The corresponding node stores the information in its Binding Cache. Then directly the communication take place which makes it insecure as there is no authentication mechanism between $\mathrm{MN}$ and
$\mathrm{CN}$. So, there may be a connection hijacking attack take place. [26]

- Connection hijacking: It is a type of attack where the attacker attacks the host client and retrives data from it The attacker can intercept packets and also can redirect packets.

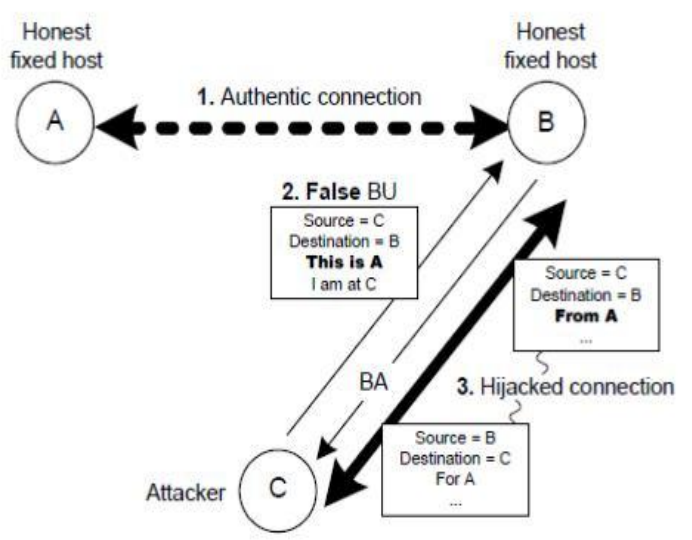

Fig 11:Connection Hijacking Technique (source:google images)

- Denial of services: It is a type of attack where the attacker makes an attempt to make the resources unavailable to the user.It is the effort to prevent to access multiple websites from a computer.

- Eavesdropping: It means theft of information from a computer.It may be of two types:

- Passive: It happens when an attacker starts to listen to the traffic and get useful data by gathering the data session and recording the data transfer between the mobile node and its HA.

- Active:Here, the attacker connects with the victim and relays message between them.It threatens the integreity and availability of the system. It may harm the system or may alter the data. [26]

\section{Solution}

The current status of MIPv6 has a lot of security issues. These issues can be solved by using a cryptogtographic algorithm for both encryption and decryption. In this paper $\mathrm{i}$ am proposing to use assymetric key algorithm. In assymmetric key cryptography we use 2 keys, one public key and one private key.If a communicating party, $\mathrm{A}$ wants to communicate with another party, B, A will first encrypt a message using B's public key and B decrypts it using A's private key. Thus, it can provide protection in terms of authentication, confidentiality and key exchange.

\section{DIFFERENCE OF MIPV4 AND MIPV6}

- MIPv6 are tunnelled using an IPv6 routing header rather than IP encapsulation, whereas MIPv4 uses encapsulation for all packets. [5]

- The dynamic HA address discovery mechanism in IPv6 returns a single reply to the mobile node. In the directed broadcast approach, which is used in IPv4, returns separate reply from each HA. [2]

- Mobile IPv6 uses destination options which allows the IPv6 control traffic to transmit backwards on any 
existing IPv6 packets, whereas MIPv4 and its route optimization extensions needs separate UDP packets for each control packets. [2]

- A key design point of MIPv4 was to support host mobility in network without mandating changes to every existing IPv4 node, but MIPv6 includes exclusive support for host mobility. [5]

- In MIPv4 special routers is required as "Foreign Agent", which is not required in MIPv6. [5]

\section{CONCLUSION}

In this paper the technical details of the protocol, status of its standardization processes, various problems associated to it, are explored. It is also discussed that how a Mobile IP allows a user to roam transparently from place to place within the internet. The various advertisement and registration protocols are described in detail.

Mobile IP plays and will play a very important part in mobile networking in future. Currently there are two versions of Mobile IP namely MIPv4 and MIPv6. MIPv6 deals with next generation internet. In this paper, the performance and working principle of these protocols are also have been studied.

In this paper, a security issues mobile IPv6 is also proposed.

I hope that brief introduction to mobile IP will engender interest in the solution to the remaining problems which continue to challenge deployment of the protocol. Future work is addressing new mechanisms to provide quality of service support while maintaining the same simple lightweight protocol approach to host mobility and wireless access to the internet. [12]

\section{REFERENCES}

[1] C.Perkins, "IP Mobility support", Network Working group, RFC 2002,Oct 1996

[2] D. Johnson and C.Perkins, "Mobility support in IPv6," draft-ietf-mobileip-ipv6-03.txt, Nov 1996

[3] D. Johnson and C.Perkins, "Route optimization in Mobile IP," IETF , November 1997

[4] J. Cao, L. Zhang, H. Chan, "Design and performance evaluation of an improved mobile IP Protocol", IETF

[5] Fayza Nada, "Performance analysis of Mobile IPv4 and Mobile IPv6," IETF, March 2006

[6] Tran Cong Hung, "Research Handover on Mobile IP" cyber journals ,July 2012

[7] Amit Mahajan, Ben Wild, "Route Optimization in Mobile IP"'

[8] G. Chellani, A Kalla, "A Review :Study of Handover Performance in Mobile IP," IJCNC, Nov 2013
[9] Nareshkumar R Mustary and Dattatraya T.H, "Reinnovation and comparison of Mobility Approaches for Mobile IP Networks"

[10] Khaled Mahmood Al-Adhal, Dr. S.S Tyagi "Mobile IP: A study of isues, challenges, and comparision of IPv4 and Ipv6," IJERA, nov-dec 2012

[11] Amit Gupta, Sumit Miglani, Maninder Singh, "Analysis of Mobile IP Protocols Security"

[12] AndrasG.Valko, Andrew T. Campbell "Cellular IP," Columbia University, New York

[13] J.Redi, P.Bahl "Mobile IP: A solution for transparent, seamless mobile computer communications" Report on upcoming trends in mobile computing and communication, 1998

[14] J. Solomon "Mobile IP: The Internet Unplugged," Prentice Hall, Englewood Cliffs, New Jersey, USA, 1998(ISBN:0-13-856246-6)

[15] Wei Wu, WenShuing Chen, Ho-En Liao and Fongray Frank Young, "A seamless handoff approach of Mobile IP Protocol for mobile wireless data networks"

[16] XiuJia Jin, "A survey on Network Architecture for Mobility"

[17] YoungsongMun and Hyewon K. Lee "Understanding IPv6", Soongsil University, Seoul, Korea; Daejin University, Kyungki, Korea

[18] Sun microsystems, "Mobile IP Administration Guide" part no-806-6542-10, January 2001

[19] Jin-Woo Jung, Hyun-Kook Kahng, RanganathanMudumbai, Doug Montgomery, "Performance Evaluation of Two Layered Mobility Management using Mobile IP and Session Initiation Protocol"

[20] Sumit Kumar, Anil Kumar, Vinay Kumar Nigam, Rakesh Kumar, "Perceptive approach for route optimization in mobile ip"

[21] C. Perkins, "IP Mobility Support for IPv4"

[22] Charles perkins, sun microsystems, "MOBILE IP"

[23] Oracle, "System Administration Guide"

[24] Sumit Kumar, Anil Kumar, Vinay Kumar Nigam, Rakesh Kumar, "Perceptive approach for route optimization in mobile ip"

[25] Dr. Rocky K.C Chang, "An Introduction to Mobile IPv4", 12 March,2002

[26] Vishwajit K. Barbudhe, Aumdevi K. Barbudhe, "Mobile IPv6: Threats and solution", IJAIEM, vol-2, Issue-6, June 2013 